\title{
Single suture customized loop for large iridodialysis repair
}

\author{
This article was published in the following Dove Press journal: \\ Clinical Ophthalmology \\ 28 September 2016 \\ Number of times this article has been viewed
}

\author{
Mohamed Omar Yousif \\ Department of Ophthalmology, \\ Faculty of Medicine, Ain Shams \\ University, Nasr City, Cairo, Egypt
}

Correspondence: Mohamed Omar Yousif Department of Ophthalmology, Faculty of Medicine, Ain Shams University, 6 Ismail Al-Kappani Street, Zone I, Nasr City, Cairo, Egypt

Tel +20 100 I68 209l

Email momaryo@hotmail.com

\begin{abstract}
Managing large iridodialysis that may occur during phacoemulsification is challenging. I describe how a procedure to reposit a prolapsed iris while the anterior chamber is markedly inflated by a current of infusion fluid may inadvertently lead to large iridodialysis, and discuss how to avoid such a complication. I describe a fast and efficient technique for managing large iridodialysis both immediately, once it occurs, or as a secondary maneuver. My technique involved fixing the iris periphery back to its root at the anterior chamber angle using 10-0 polypropylene suture with two straight needles introduced directly through the cornea at distant points, and an insulin syringe as a guide track to a point $1.5 \mathrm{~mm}$ from the limbus at the base of a triangular scleral flap that was designed to be centered on the area of iridodialysis. I confirmed the simplicity, efficacy, and safety of my technique through a 1-year follow-up period.
\end{abstract}

Keywords: iridodialysis, iridoplasty, pupilloplasty, phaco complications

\section{Introduction}

Iridodialysis is not an uncommon complication during phacoemulsification procedures or any intraoperative maneuver, since the iris root is the thinnest and weakest portion of the stroma. When the eye is contused or the iris is accidentally engaged during an operation, separation occurs most frequently at this site. ${ }^{1}$ Superior defects covered by the upper eyelid may be less symptomatic; however, an iridodialysis in any other quadrant can result in potentially debilitating visual sequelae, including monocular diplopia, glare, and photophobia. In the pseudophakic state, corectopic pupils can potentially expose the edge of an intraocular lens, with the attendant undesired optical phenomena. ${ }^{2}$ Iridodialysis may also cause cosmetic problems. For all these reasons, such conditions must be treated properly. ${ }^{3}$

Several previously described techniques have addressed ways for management of such a problem; however, complications of inappropriate management can be disastrous, making skilled repair a must and the search for novel techniques an ongoing project.

Complications vary widely; for example, inadvertent engagement of the ciliary body causing hemorrhage or increased cyclitis, pulling the iris tissue more peripherally than its initial origin causing corectopia, and if the suture is tightened and the iridodialysis is reduced to the scleral wall, the iris within the suture bite, not the actual iris root, is reapproximated near the iris base, leaving less stroma between the pupil margin and the scleral wall. Corectopia can be further exaggerated by the fibrotic response that commonly occurs in the band of disinserted iris stroma. Furthermore, the disinserted sector of sphincter muscle is deprived of its radial innervation and blood supply and 
thus has reduced tone that acts to counteract the curling up of this delicate iris tissue. ${ }^{2}$ Techniques described to repair iridodialysis can be classified into two groups: open chamber and closed chamber techniques. ${ }^{4}$ Open chamber techniques access the iridodialysis site through a limbal self-sealing incision (one of the phaco incisions) or a scleral tunnel incision. ${ }^{5}$ On the contrary, access to the anterior chamber (AC) is attained with a needle in closed chamber techniques. ${ }^{4}$ In the continuous search for new, less traumatizing, and more physiological ways for repair of iridodialysis, I hereby present my technique, an emergency technique that has the advantage of the ability to be used intraoperatively, if such a complication occurred during any intraocular surgery, and that can be used as a second-step procedure for any cold case having iridodialysis.

This technique has proved validity, efficacy, as well as anatomical and physiological satisfactory results.

\section{Surgical technique}

My technique was first used in an emergency situation (Video 1), where during a phacoemulsification case in a 50-year-old patient with faint posterior subcapsular cataract, the patient's iris was severely traumatized due to several maneuvers trying to reposit a prolapsed iris, with the irrigation probe still inside the $\mathrm{AC}$, raising the intracameral pressure and increasing the prolapsed tissue, while the surgeon was trying to reposit the iris in the face of such a prolapsing power, and that was totally opposing laws of nature and physics of fluidics (Figures 1A and 2A).

Such iris traumatization resulted in a large iridodialysis, extending nearly the whole vertical $180^{\circ}$ (Figures $1 \mathrm{~B}$ and $2 \mathrm{~B}$ ), necessitating a rapid innovative repair technique; by this time, the patient still had remaining unremoved epinucleus and cortex, and an intraocular lens to be implanted.

My technique involved fixing the iris back to its root using a double-armed 10-0 polypropylene suture with two straight needles (STC-6; Ethicon, Somerville, NJ, USA). Conjunctival peritomy centered over the area of iridodialysis was performed, followed by cauterization of the underlying scleral bed around any broken blood vessels.

A partial thickness triangular scleral flap centered over the dialysis area was then performed using a crescent knife (Figures 1C and 2C).

Introduction of the first needle was not done through the main phaco wounds, so as to be able to control entry sites allowing proper accessibility of these two faraway ends of the large iridodialysis area, and also to avoid any inadvertent trauma to intraocular structures, such as the bare posterior capsule.
The first needle was introduced through the patient's cornea in a way resembling McCannell suture opposing the first end of the dialysis. A 27-gauge needle was introduced below the scleral flap at the proposed anatomical site of the iris root, which was $\sim 1.5 \mathrm{~mm}$ posterior to the limbus, consistent with the landmark adopted by Agarwal et $\mathrm{al}^{6}$ as an external anatomical landmark for the iris root. The direction of needle passage also affects significantly where the needle will come out inside the eye, and so the 27 -gauge needle was used to help guide the straight needle out of the AC avoiding any possible injury and making sure it comes out at a point $1.5 \mathrm{~mm}$ posterior to the limbus (Figures 1D and 2D).

The second polypropylene needle was then also passed through the cornea facing the other end of the large iridodialysis and through iris periphery and guided outside to the base of the scleral flap using an insulin syringe. Now, with the two needles coming out below the scleral flap, two polypropylene threads will be passing through the AC (Figures 1E and 2E).

These two threads were then withdrawn through the main phaco wound using a Sinskey hook forming two loops arising from the main wound (Figures $1 \mathrm{~F}$ and $2 \mathrm{~F}$ ).

Cutting these two loops (Figures $1 \mathrm{G}$ and $2 \mathrm{G}$ ) and tying the cut ends together (Figures $1 \mathrm{H}$ and $2 \mathrm{H}$ ) gives rise to a single loop whose two ends are now passing through the two ends of the dialysis and coming out right below the scleral flap. Holding the iris tissue with this loop allows full control on iris, repositioning back to its root, and full ability of suture tension control (Figures 1I and 2I).

The knot is then extruded to outside the eye by pulling on one of the two sutures (Figures $1 \mathrm{~J}$ and $2 \mathrm{~J}$ ), and then, pulling on the other suture in the opposite direction is performed (Figures $1 \mathrm{~K}$ and $2 \mathrm{~K}$ ) to correct any wrinkles in the iris periphery that might have occurred by the first pull, aiming at inhibition of any form of corectopia.

The free ends of the suture are then tied (Figures 1L and $2 \mathrm{~L}$ ), and the scleral flap is then sutured with single nylon $10-0$ suture (Figures $1 \mathrm{M}$ and $2 \mathrm{M}$ ), with the knot now below it.

Phacoemulsification was then completed with removal of the remaining lens matter, hydration of the main wounds, and closure of the main phaco wound with a single buried nylon 10-0 stitch for safety.

Conjunctival peritomy was cauterized, avoiding stitches so as to minimize postoperative patient discomfort.

This technique proved a huge success with postoperative visual acuity of 20/20 only 1 week after, and with near absence of all feared complications of iridodialysis repair; there was no increase in the patient's intraocular 

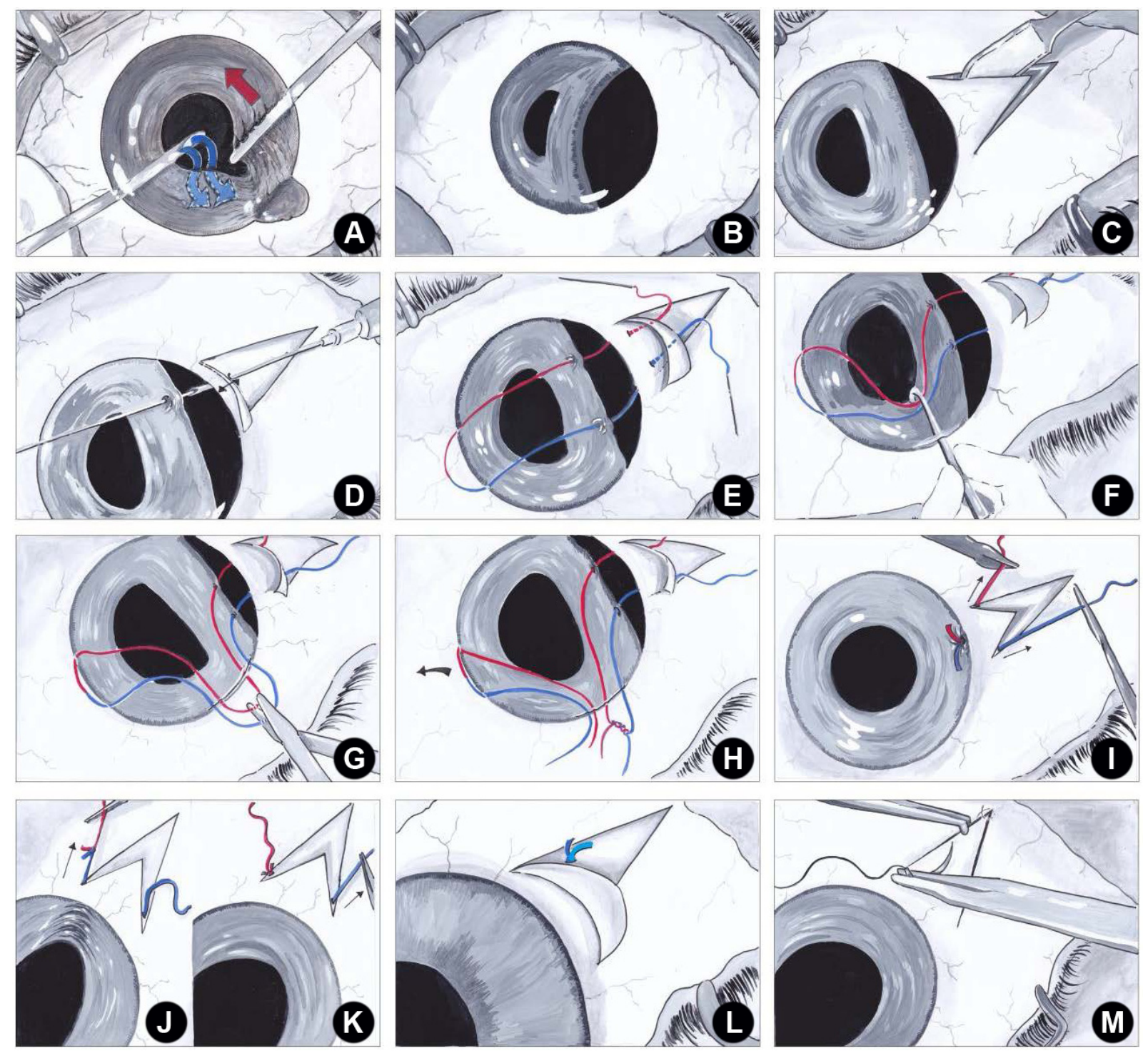

Figure I Illustrative representation of (A) the wrong way of iris repositioning using the spatula against the irrigation fluid current; (B) large iridodialysis extending throughout the vertical $180^{\circ}$; (C) creation of partial thickness triangular scleral flap centered over the dialysis area using a crescent knife; (D) introduction of the first straight needle of a double-armed polypropylene suture through the cornea and then through one end of the dialysis, and then using a 27 -gauge needle introduced below the scleral flap I.5 mm posterior to the limbus as a guide track; $(E)$ introduction of the second polypropylene suture straight needle through the cornea facing the other end of the huge dialysis; (F) withdrawing the two threads from the AC through the main phaco wound using a Sinskey hook, forming two loops arising from the main wound; (G) cutting of the two loops; (H) tying the cut ends together; (I) pulling on the two ends of the polypropylene loop from beneath the scleral flap to reposit the iris back to its root; $(\mathbf{J})$ pulling on one of the two suture ends to extrude the knot from the AC; $(\mathbf{K})$ pulling on the other suture in the opposite direction to correct any wrinkles in the iris periphery that might have been caused by the first pull; (L) tying the free ends of the sutures below the scleral flap; and (M) suturing the scleral flap with single nylon I0-0 suture at the apex.

Abbreviation: AC, anterior chamber.

pressure (IOP) and only minimal uveitic reaction in the first postoperative day with round regular pupil shape and perfect centration, denying any possible corectopia or irregularity. Gonioscopy revealed pigment dispersion only on the trabecular meshwork with no peripheral anterior synechia.

Figure 3 demonstrates intraoperative photographs of the eye before and after repair.

Being encouraged by such results and in pursuit of trying to prove this technique as a valid and completely safe technique for iridodialysis repair, another case was subsequently managed using the same exact technique, but this time in a cold case of iridodialysis that occurred during a phacoemulsification in a 64-year-old patient, that was not managed intraoperatively, and was 1 week old. The same satisfying results were obtained except only for the presence of $<1$ hour peripheral anterior synechia on gonioscopy. These amazing results helped in proving the validity of the procedure.

Both patients received the usual postoperative treatment of prednisolone acetate eye drops $1 \%$ qid and topical gatifloxacin $0.3 \%$ five times per day. They were followed up for 1 year with the aim of exclusion of any late complications that might 

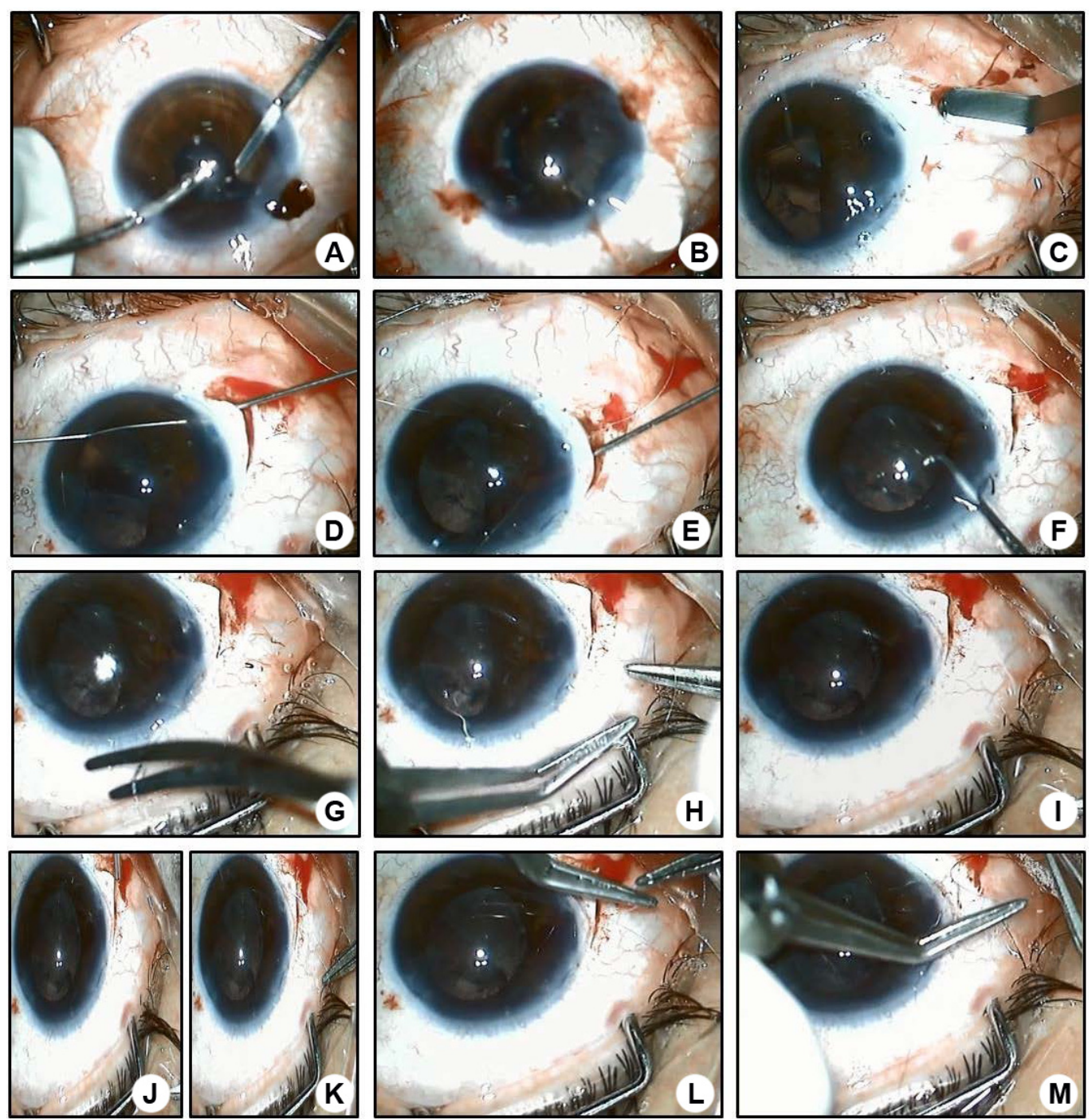

Figure 2 Photographic representation of (A) the wrong way of iris repositioning using the spatula against the irrigation fluid current; (B) large iridodialysis extending throughout the vertical $180^{\circ} ;(\mathbf{C})$ creation of partial thickness triangular scleral flap centered over the dialysis area using a crescent knife; (D) introduction of the first straight needle of a double-armed polypropylene suture through the cornea and then through one end of the dialysis, and then, using a 27-gauge needle introduced below the scleral flap $1.5 \mathrm{~mm}$ posterior to the limbus as a guide track; $(E)$ introduction of the second polypropylene suture straight needle through the cornea facing the other end of the huge dialysis; (F) withdrawing the two threads from the AC through the main phaco wound using a Sinskey hook forming two loops arising from the main wound; (G) cutting of the two loops; (H) tying the cut ends together; (I) pulling on the two ends of the polypropylene loop from beneath the scleral flap to reposit the iris back to its root; (J) pulling on one of the two suture ends to extrude the knot from the AC; $(\mathbf{K})$ pulling on the other suture to the opposite direction to correct any wrinkles in the iris periphery that might have occurred by the first pull; (L) tying the free ends of the sutures below the scleral flap; and (M) suturing the scleral flap with single nylon I0-0 suture at the apex. Abbreviation: AC, anterior chamber.
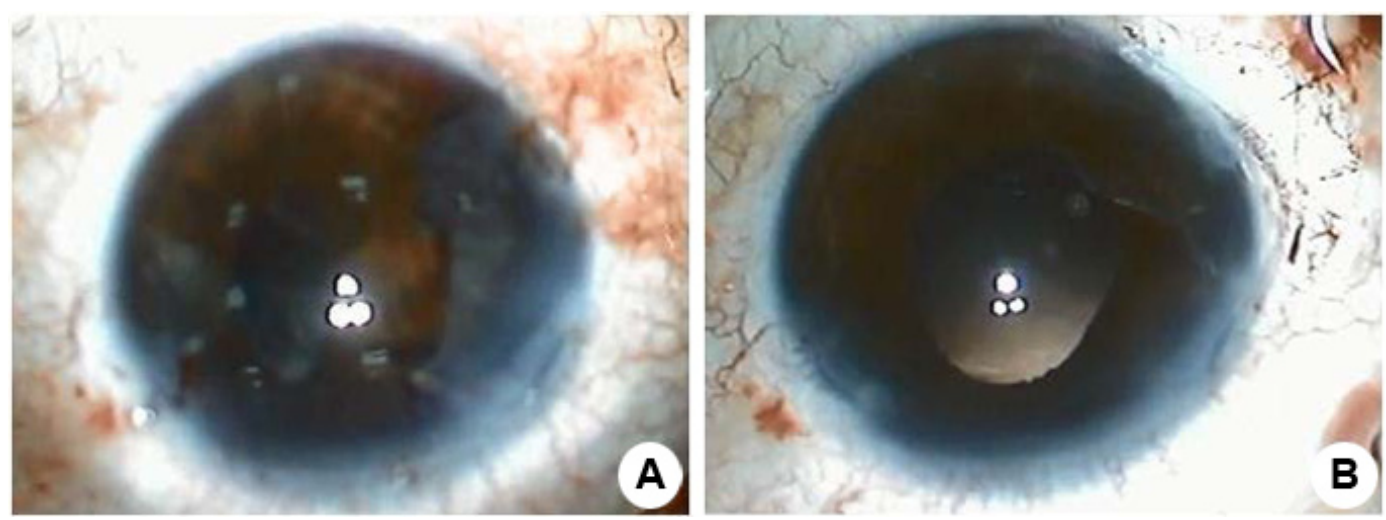

Figure 3 Photographs of the eye before (A) and after (B) repair. 
have not appeared during the early postoperative period. Follow-up visits were at 1 week, 1 month, 3 months, 6 months and 1 year interval with the assessment of uncorrected visual acuity (UCVA), best-corrected visual acuity (BCVA), IOP, full anterior segment slit lamp examination, fundus examination, and gonioscopy for the angle of the AC.

For the first patient, the UCVA and BCVA were 20/20 at all follow-up visits, mild $\mathrm{AC}$ reaction was noticed on the first postoperative day, the IOP ranged between $15 \mathrm{mmHg}$ and $18 \mathrm{mmHg}$ in all visits, and gonioscopy done at 3 months and 6 months postoperatively revealed pigment dispersion only on the trabecular meshwork with no peripheral anterior synechia.

For the second patient, the UCVA was 20/60 that corrected to only $20 / 40$ on the first postoperative day. I suggested that the defective vision was due to the moderate-to-severe $\mathrm{AC}$ reaction caused by the delayed repair and not by the maneuver itself; the BCVA was 20/20 at the remaining follow-up visits. The IOP ranged between $16 \mathrm{mmHg}$ and $20 \mathrm{mmHg}$ at all visits, and gonioscopy performed at 3 months and 8 months postoperatively revealed pigment dispersion over the trabecular meshwork, in addition to a band of peripheral anterior synechia taking only 1 hour.

\section{Discussion}

During the ongoing pursuit for newer surgical techniques in all ophthalmological aspects, emergency management techniques remain a priority. Several previous techniques have been introduced for iridodialysis repair. I hereby might have achieved advantages over most of these previous techniques, not only by the technique itself, but also because it can be used as a rescue measurement if such a complication occurred during phacoemulsification or any kind of intraocular surgery.

Although my technique might seem to be a little bit complicated at first, once mastered and performed even a single time, it is much easier to perform and much less time consuming compared with most other techniques.

Bardak et al, ${ }^{3}$ for instance, introduced a technically challenging procedure for closed chamber iridodialysis repair using a needle with a distal hole, where the technique involved 10-0 polypropylene suture attached to the hole at the tip of the needle. The needle was inserted into the AC from the point of initial entry; it was pushed forward to $\sim 1.0 \mathrm{~mm}$ behind the separated iris tissue. It was then pushed through the $\mathrm{AC}$ and pulled out through the sclera $\sim 1.0 \mathrm{~mm}$ behind the limbus. One loop of the suture was taken off the needle, while the second loop was still attached. The needle was pulled back into the $\mathrm{AC}$, and the procedure was repeated using a different needle two to four times depending on the size of the iridodialysis. ${ }^{3}$

My technique is a single-pass technique as mentioned, making it easy, fast, and less traumatizing to the iris, sclera, and cornea. The technique also has the advantage of burying the final polypropylene knot below the scleral flap, unlike in the study of Bardak et al, ${ }^{3}$ where their knot was either rotated into the iris tissue or left sub-conjunctivally according to their estimated iris status. If the iris status allowed such a knot rotation, the rotation increases the incidence of corectopia; if the iris condition did not allow such a rotation, the knot was left directly subconjunctival, which can significantly increase the risk of subconjunctival bleeding - this actually occurred in a few of their cases. This technique also increased the postoperative hyphema, chemosis and, surprisingly, iridodialysis appeared in one of their patients postoperatively. ${ }^{3}$

Khokhar et $\mathrm{al}^{7}$ described what they called the stroke and dock technique for iridodialysis repair. Their technique involved a $2.75 \mathrm{~mm}$ limbal incision $180^{\circ}$ opposite to the center of the dialysis to introduce two double-armed polypropylene suture needles through the central area of the periphery of the iris dialysis, and guide them outside to the base of scleral flap using a 27-gauge syringe. Although this technique is simple and carries the same concept as my technique, I tried to avoid the restriction of the entry site to a limbal incision that is $180^{\circ}$ opposite to the iridodialysis point, which might hinder the ergonomic movement needed to direct the needle to where the surgeon suggests as an ideal position of peripheral iris bite, especially if the iridodialysis is large.

My technique offers the flexibility of what the surgeon sees as more suitable for any entry site, and allows the 10-0 polypropylene suture needle to enter easily and engage the two points at the dialyzed iris periphery, even if way below or above the horizontal $180^{\circ}$. This can be case-tailored or customized according to what fits the situation and to where sutures are supposed to produce a more regular contour. The procedure was performed without being obliged to introduce the two needles of the double-armed polypropylene suture through the same limbal incision, and hence, restricting iris sutures to points that are too close to each other and are $180^{\circ}$ opposite to that incision.

Despite repairing such dialysis as an emergency, I did not use intracameral miotics. This is in contrast to what was done by Snyder and Lindsell ${ }^{2}$ where, despite being a cold case of repair, intraocular carbachol was injected into the $\mathrm{AC}$ in a trial to cause iris stretch. I tried to avoid injecting 
miotics to minimize postoperative uveitic reaction that might be induced by them. Additionally, I still had a phaco case to complete where injecting a miotic would not allow for the proper visualization or removal of the remaining epinuclear or cortical matter, or implantation of an intraocular lens. Snyder and Lindsell ${ }^{2}$ also implied that multiple sutures might be needed to repair a larger dialysis than what they presented or any dialysis of large size. On the contrary, in my technique, I was already challenged by the first case of large iridodialysis, and the idea of my loop successfully repairing such a large dialysis ( $~ 5$ hours) by only one knot, two guided passes and one loop would, in my judgment, cause unnecessary traumatization of delicate ocular structures.

Snyder and Lindsell ${ }^{2}$ also adopted the idea of rotating the knot into the scleral wall. This caused a centripetal displacement of the pupil that they stated was temporary but might be permanent if the iris was tightly secured to the scleral wall.

The idea of a suture left in the AC was suggested by Özdek and Özmen ${ }^{4}$ as a solution, instead of leaving the irritating knot unburied or directly below the conjunctiva.

As I described in my technique, there are two opposite pulls on the two ends of the loop: the first pull aims at knot extrusion from the $\mathrm{AC}$, while the second and opposite pull on the other end of the loop allows for removal of any peripheral iris wrinkles that might have occurred from the first pull. This maneuver results in absence of a knot in the AC, avoiding unnecessary chronic iris irritation. At the same time, the idea of having two successive pulls in two opposite directions results in the advantage of total absence of corectopia with rounded, regular, and fully reactive pupils.

I also adopted the suggestion of Agarwal et al, ${ }^{6}$ stating the most anatomically suitable position for the scleral exit of the 10-0 polypropylene suture straight needle, which is to be at $1.5 \mathrm{~mm}$ posterior to the limbus. In order to prove the validity of such a hypothesis, two patients had gonioscopic examination at every single visit throughout their 1-year follow-up period, which showed only pigment dispersion over the trabecular meshwork (as mentioned earlier), with $<1$-hour peripheral anterior synechia in the second patient and no recorded elevation of the IOP in both cases.

As far as we know, trials for repair of the dialysis (once it had occurred intraoperatively, not as a second-step maneuver), have not, up until now, clearly established a suitable and widely accepted technique, except for a single mention by Oshika et al. ${ }^{8}$ Even then, the technique was not fully described, as they mentioned just one case report, that included only a 1-month follow-up period with no adequate record of complications thereafter.
From my first case, and apart from the repair itself, I definitively conclude that, as stated earlier, the iris is one of the most sensitive and delicate intraocular structures and there is always a right way to deal with it. I can introduce what appears to be an effective method to use whenever an iris gets prolapsed. First and foremost, we always have to decrease IOP and intracameral pressure, not push against a push, or against high positive pressure, as it is not wise trying to reposit a prolapsed tissue while placing the irrigation probe inside. This only increases tension and traumatization, and might end in a disastrous iridodialysis.

Beside simplicity, reproducibility, and safety, we can summarize the added value of my technique over previously used techniques in three main points: first, the use of customized entry points through the corneal periphery to access customized peripheral locations of the dialyzed iris tissue, using two guided passes and single polypropylene suture, allows better and more precise anatomical apposition and more tension control without excessive manipulation of the delicate iris tissue; second, the knot extrusion outside the AC results in less chronic iris irritation and induced uveitis; third, the further suture pull in the opposite direction of knot extrusion treats any induced corectopia.

\section{Acknowledgments}

The author would like to acknowledge the role of Eman Samir Edrees, MD, assistant lecturer at Ain Shams University for her valuable assistance in formulation and revision of the article. This surgical technique was presented at the American Society of Cataract and Refractive Surgery (ASCRS) 2016, New Orleans, LA, USA, on May 8, 2016. The video describing the surgical technique was presented and won the People's Choice Awards - runner-up in ASCRS 2015 Film Festival, San Diego (title: Managing Large iridodialysis during Phacoemulsification).

\section{Disclosure}

The author reports no conflicts of interest in this work.

\section{References}

1. Kumar S, Miller D, Atabara NA, Blance E. A quantitative animal model of traumatic iridodialysis. Acta Ophthalmol (Copenh). 1990;68(5): 591-596.

2. Snyder M, Lindsell L. Nonappositional repair of iridodialysis. J Cataract Refract Surg. 2011;37(4):625-628.

3. Bardak Y, Ozerturk Y, Durmus M, Mensiz E, Aytuluner E. Closed chamber iridodialysis repair using a needle with a distal hole. J Cataract Refract Surg. 2000;26(2):173-176.

4. Özdek S, Özmen M. A simple surgical technique for repair of iridodialysis. Turk J Med Sci. 2009;39(2):317-319. 
5. Paton D, Craig J. Management of iridodialysis. Ophthalmic Surg. 1973; 4(1):38-39.

6. Agarwal T, Singh D, Panda A. Guide needle-assisted iridodialysis repair J Cataract Refract Surg. 2011;37(10):1918.
7. Khokhar S, Gupta S, Kumar G. Iridodialysis repair: stroke and dock technique. Int Ophthalmol. 2014;34(2):331-335.

8. Oshika T, Amano S, Kato S. Severe iridodialysis from phacoemulsification tip suction. J Cataract Refract Surg. 1999;25(6):873-875. 


\section{Supplementary material}

Video I Video representing how an inadvertent way of iris repositioning using the spatula opposite to the direction of irrigation fluid current leads to a large iridodialysis extending throughout the vertical $180^{\circ}$.

Notes: This large iridodialysis was repaired by fixing the iris periphery back to its root using single double-armed polypropylene suture. I started by creation of partial thickness triangular scleral flap centered over the dialysis area using a crescent knife. This was followed by introduction of the first straight needle of a double-armed polypropylene suture through the cornea and then through one end of the dialysis and then getting it out to the base of the scleral flap at a point that was 1.5 mm posterior to the limbus using a 27-gauge needle as a guide track. The same maneuver was repeated with the other needle of the double-armed suture but with gripping the iris periphery at a point that was a bit far from the first one. The two threads were then withdrawn from the AC through the main phaco wound using a Sinskey hook forming two loops arising from the main wound. The two loops were cut, and then the cut ends were tied together. The two ends of the polypropylene loop were pulled from beneath the scleral flap to reposit the iris back to its root. This was followed by pulling on one of the two suture ends to extrude the knot from the AC and then pulling on the other suture in the opposite direction to correct any wrinkles in the iris periphery that might have occurred by the first pull. Finally, the free ends of the sutures were tied below the scleral flap and the scleral flap was sutured with single nylon 10-0 nylon suture at the apex.

Abbreviation: AC, anterior chamber.

\section{Publish your work in this journal}

Clinical Ophthalmology is an international, peer-reviewed journal covering all subspecialties within ophthalmology. Key topics include: Optometry; Visual science; Pharmacology and drug therapy in eye diseases; Basic Sciences; Primary and Secondary eye care; Patient Safety and Quality of Care Improvements. This journal is indexed on

Submit your manuscript here: http://www.dovepress.com/clinical-ophthalmology-journal

\section{Dovepress}

PubMed Central and CAS, and is the official journal of The Society of Clinical Ophthalmology (SCO). The manuscript management system is completely online and includes a very quick and fair peer-review system, which is all easy to use. Visit http://www.dovepress.com/ testimonials.php to read real quotes from published authors. 